Revista Oficial del Poder Judicial

ÓRGANO DE INVESTIGACIÓN DE LA CORTE SUPREMA DE JUSTICIA DE LA REPÚBLICA DEL PERÚ

Vol. 11, n. ${ }^{\circ} 13$, enero-junio, 2020, 117-138

ISSN versión impresa: 1997-6682

ISSN versión electrónica: 2663-9130

DOI: https://doi.org/10.35292/ropj.v11i13.41

\title{
El bloque de convencionalidad en el desarrollo del ISN que evalúa derechos de orden multidimensional $e$ interdependientes \\ a favor de niños y adolescentes
}

Standards of the constitutional block to

determine the Best Interests of the Child

from a multidimensional and interdependent

perspective fauoring children's rights

(우웅

MANUEL BERMÚDEZ-TAPIA

Universidad Nacional Mayor de San Marcos

Universidad Privada San Juan Bautista

(Lima, Perú)

Contacto: mbermudezt@unmsm.edu.pe

manuel.bermudez@upsjb.edu.pe

http://orcid.org/0000-0003-1576-9464

\section{RESUMEN}

En la evaluación de los conflictos sociofamiliares que registran un trámite en el Poder Judicial, se destaca una situación particular: la tutela de los derechos de personas dependientes, con una minoridad de edad, que están sometidas a la discrecionalidad de sus progenitores y que no logran ser atendidos en sus necesidades 
más básicas y urgentes (alimentos), especialmente porque el trámite judicial no evalúa la verdadera dimensión de sus derechos, que en esencia se convierten en multidimensionales e interdependientes, y sobre la cual se requiere reconfigurar la naturaleza jurídica del interés superior del niño para así desarrollar una mejor definición que parta de la defensa del derecho alimentario y se extienda a otros derechos de niños y adolescentes.

Palabras clave: derechos de alcance multidimensional, derecho de alimentos, metaprincipio jurídico, interés superior del niño, tutela judicial efectiva y debido proceso, conflicto familiar, proceso de alimentos.

\section{ABSTRACT}

Statistics of the Judicial system reveal some social and familiar conflicts relevant to the study. One of them is child support. Child support needs to be construed according to the constitutional block standards. That interpretation includes the principle of Best Interests of the Child and the multidimensional and interdependence values to safeguard children's rights. Finally, there is a need to apply the same criteria to other legal regulations like juvenile's rights for example.

Key words: constitutional block standard, children's rights, multidimensional, independence.

Recibido: 25/05/2020 Aceptado: 10/06/2020

\section{INTRODUCCIÓN}

La evaluación de un conflicto familiar judicializado (Thomlison, 2010, p. 52) permite analizar una serie de elementos que registran un contexto en el cual se complementan:

a) Una deficiente evaluación del derecho evaluado por parte de todos los involucrados (Fortin, 2005, p. 285). 
b) La ampliación de la violencia en el ámbito temporal.

c) La interdependencia del derecho alimentario con otros derechos posibilita detallar su carácter flexible en complementación con otros derechos; por este motivo lo consideramos el elemento «articulador» que propicia su evaluación multidimensional.

d) Las limitaciones del Sistema de Impartición de Justicia de no poder evaluar diligentemente un expediente en el cual se analizan los derechos de un niño o un adolescente.

En función de ello, el elemento que origina esta evaluación parte del análisis del derecho alimentario porque este es el que usualmente ayuda a visualizar el conflicto familiar en el ámbito judicial y sobre el cual se plantean otros procesos judiciales o acciones que involucran denuncias, por registro de alguna situación de violencia, ante la Policía Nacional o el Ministerio Público (Bermúdez-Tapia, 2011b, p. 39).

De acuerdo con estos alcances, el interés superior del niño (Aguilar, 2008) parecería un elemento impreciso que no faculta el análisis de un caso en forma diligente, de modo que se generan deficiencias en varios ámbitos y niveles, desde lo dogmático hasta lo judicial, desde lo sustantivo hasta lo procedimental, desde lo nacional hasta la práctica judicial convencional (Burgorgue-Larsen y Úbeda, 2011, p. 397), dado que el alimentista no podría defender su derecho porque no es «parte procesal».

\section{EL NIÑO Y EL ADOLESCENTE COMO SUJETOS DE DERECHOS EN EL CONFLICTO FAMILIAR JUDICIALIZADO DEL MENOR}

Los niños y los adolescentes que participan en un conflicto familiar judicializado ven condicionados sus derechos en las siguientes situaciones:

a) Cuando están en un contexto de ausencia de un progenitor o se hayan inmersos en una realidad familiar múltiple, porque 
la atención de sus necesidades es limitada por una acción ajena a su realidad y comprensión (Hewitt-Taylor, 2008, p. 30).

En este sentido, las familias monoparentales, convivenciales impropias, concubinarias o paralelas provocan de modo automático una condición que afecta el desarrollo del niño o adolescente, quien asume una condición psicológica de «normalidad» de una experiencia que, en sí misma, es extraordinaria y negativa.

La evaluación de la condición de abandono material y moral resultaría, inclusive, una situación compleja de evaluar, especialmente cuando se confunden los niveles de pobreza con respecto de los niveles de atención de las necesidades básicas de los hijos; la mejor evidencia para acreditar este punto está expuesto en el caso Ramírez Escobar y otros vs. Guatemala (Corte Interamericana de Derechos Humanos, 2018).

b) Cuando el conflicto familiar entre los progenitores ha superado el contexto privado puede producir la desatención de los derechos de los niños y los adolescentes, lo que no necesariamente genera una acción en el ámbito judicial, debido a la procrastinación del progenitor que asume la tenencia, generalmente ficta.

En este punto, los progenitores asumen comportamientos negligentes y negativos en detrimento del cuidado de su propio hijo; dicho comportamiento es percibido en varios niveles: desde la negligencia hasta el acto de sometimiento psicológico hacia un progenitor que ejerce una posición de dominio y provoca el síndrome de Estocolmo en el otro progenitor (Bermúdez-Tapia, 2019a, p. 196).

En esta primera etapa, el niño o adolescente es afectado por la acción directa de sus propios progenitores, quienes 
actúan tanto en forma activa como pasiva en la ejecución, de modo contrario a la obligación moral y civil de velar por su integridad y desarrollo.

c) Cuando el conflicto familiar se ha judicializado, los progenitores, los magistrados y todos aquellos que participan en el proceso judicial (peritos, asistentes sociales, policías, etc.) no logran comprender la situación de violencia que afronta el niño o el adolescente al estar sumido en un conflicto que supera sus expectativas y nivel de comprensión.

En este ámbito, se manifiesta una condición que afecta su desarrollo psicológico en mérito de considerarse "parte del problema» o «causante» de este, lo cual puede provocar una reacción suicida (Polaino-Lorente, 1988, p. 124).

Daniel Krauss y Bruce Sales (2001) exponen una estadística que registra al $40 \%$ de niños y adolescentes afectados por estar incluidos en un conflicto familiar (en cualquier nivel e instancia), hecho que revela el elevado nivel de invisibilidad que se genera contra este grupo vulnerable y en situación de riesgo por parte de los adultos que deciden y evalúan sus derechos (p. 411).

d) Cuando el proceso judicial ha finalizado y los progenitores continúan efectuando actos de violencia entre sí, el niño o el adolescente inmerso en un conflicto temporalmente extendido asume una posición extrema respecto de sus progenitores, y puede desarrollar un patrón de alienación parental o de manipulación a sus progenitores (síndrome del niño emperador).

En este ámbito, factores exógenos al niño o al adolescente pueden provocar una condición negativa si el entorno familiar sigue en una situación de crisis, limitada o sometida a cuestiones legales que afectan a los progenitores (International 
Human Rights Law Clinic, Chief Justice Earl Warren Institute on Race, Ethnicity and Diversity \& Immigration Law Clinic, 2010, p. 12).

Y en cada una de estas etapas, su condición de «sujeto de derechos» se ve condicionada a factores ajenos a su voluntad y margen de acción, especialmente porque no posee la capacidad ni las condiciones para ejercer una tutela de sus propios derechos o no puede atenuar el nivel de conflicto entre sus progenitores. Por tanto, surgen condiciones que se amplían, en términos negativos, cuando:

a) El conflicto se extiende en el ámbito temporal: el daño psicológico que pudiera asumir un niño o un adolescente es un factor que no suele evaluarse por los progenitores cuando se separan, se divorcian o desarrollan actos de violencia entre sí y puede condicionar su comportamiento adulto (Sheehan, 2018, p. 88).

b) El nivel de la violencia física se incrementa: en este contexto, la omisión de la obligación alimentaria se convierte en un factor secundario frente a la necesidad de tutelar la integridad del progenitor que desarrolla la tenencia (generalmente ficta) y, por ello, las acciones de exigencia, reclamo o petición de alimentos no son prioritarias.

c) El conflicto se agudiza por la acción de los progenitores al desarrollar nuevos contextos familiares, luego de la separación o el divorcio o, eventualmente, un progenitor ha desarrollado dos contextos familiares en forma paralela (Bermúdez-Tapia, 2017), un detalle que implica una relación directa con la prestación alimentaria, en especial porque los obligados a proporcionarla suelen exponer como argumento para justificar su acción el hecho de que la contraparte no 
ejecuta diligentemente la atención alimentaria a los hijos. Las referencias más comunes de ello son las siguientes:

- El hecho de que la crianza de los hijos esté a cargo de los abuelos.

- El registro de condiciones negativas que limitan la prestación alimentaria porque el obligado asume nuevas cargas familiares.

- El registro de una convivencia del otro progenitor con una tercera persona a la cual identifican como un «beneficiario» de la cuota alimentaria que debería ser destinada a favor de sus hijos, con lo cual surge la denominación de «polizonte económico» (Bermúdez-Tapia, 2011a, p. 347).

De acuerdo con estas situaciones, en forma general, afirmamos que la condición de «sujeto de derechos» es un elemento nominal pero no práctico en el registro y estudio de conflictos familiares, inclusive judicializados.

\section{3. ¿LA DELIMITACIÓN DE LA NATURALEZA JURÍDICA DEL INTERÉS SUPERIOR DEL NIÑO ES UNA REGLA, UN PRINCIPIO O UN METAPRINCIPIO?}

En términos prácticos, las «reglas» evolucionan a valores de carácter objetivo, convirtiéndose en "principios jurídicos», que en algunas ocasiones provocan una deliberación respecto de su alcance, por lo cual se debe ejecutar un acto «racional de ponderación».

Al respecto, Alexander Peczenik (1983) establece dos condiciones:

a) Las reglas se aplican en la evaluación de los casos rutinarios («fáciles») porque no existe necesidad de sopesar o ponderar.

b) Sobre la base de la interpretación de los postulados de Manuel Atienza y Juan Ruiz Manero, los principios se aplican de una «manera abierta», según el caso en evaluación, porque al estar 
enunciados en términos generales pueden expresar los «valores superiores de un ordenamiento jurídico»; por ello, la libertad y la igualdad se desarrollan en función de un formato expansivo.

Por su parte, Robert Alexy (1993) refiere que los principios son normas que ordenan que algo sea factible dentro de las posibilidades jurídicas y reales existentes, por lo que son mandatos de optimización que pueden ser cumplidos en diferentes grados.

Sin embargo, pese a lo indicado, existen elementos que aún no son suficientemente objetivos en su alcance en cuanto a su optimización (Lopera, 2004, p. 213), específicamente, para analizar el interés superior del niño, ya que, desde nuestra perspectiva, este principio no logra plantear un óptimo nivel de tutela de los derechos de los niños y los adolescentes en un proceso judicial. Para analizar de manera apropiada el interés superior del niño, proponemos lo siguiente:

a) Desde él «nacen» el derecho y una obligación, como complementarios e interdependientes elementos de evaluación; debemos tener presente que el derecho alimentario - el cual, materialmente, se evalúa en primer término - se desnaturaliza por el tiempo que demora su determinación, exigibilidad y prestación en el tiempo.

b) Desde la exigibilidad y el requerimiento para el cumplimiento de derechos surgen los comportamientos evasivos, de mala fe, maliciosos y temerarios de los progenitores, que se relacionan con los actos procesales y judiciales que inician en forma paralela, complementaria, derivada o secundaria al proceso de alimentos.

c) El derecho de los niños y los adolescentes puede supeditarse a la discrecionalidad de un progenitor; esto se observa cuando no se exige su cumplimiento o se ejecuta un comportamiento negligente. 
Tomando en cuenta estos elementos, consideramos que el interés superior del niño tiene una naturaleza jurídica superior a la de una regla o un principio jurídico, pues, en esencia, su contenido debe delimitarse en un ámbito práctico y específico que, a la misma vez, es individual y excluyente de otras referencias. Basándonos en ello, preferimos identificar su naturaleza jurídica como un «metaprincipio», conforme anota José Suárez (2006), porque estos conceptos filosóficos «orientan una búsqueda creativa que genera un resultado práctico» (p. 83).

En un intento por presentar un desarrollo aplicativo de esta definición sobre la naturaleza jurídica del interés superior del niño como metaprincipio, suscribimos dos referencias equivalentes:

a) El metaprincipio de dignidad posibilita que se evalúe un caso en particular y se identifique una referencia inaplicable a otro contexto.

Esta definición y criterio de aplicabilidad proviene de la práctica jurisprudencial de la Corte Interamericana de Derechos Humanos en dos casos específicos:

- Caso Castillo Petruzzi y otros vs. Perú (1999).

- Caso Ivcher Bronstein vs. Perú (2001).

De este modo, la dignidad se evaluó en función de la gravedad de los hechos, con lo cual se acuñó una referencia "guía» del derecho convencional al determinarse que este metaprincipio se desarrolla en cuatro ámbitos específicos:

- El derecho al nombre.

- El derecho a tener una nacionalidad.

- El derecho a tener libertad de pensamiento.

- La prohibición de cualquier práctica que involucre una acción de tortura, bajo cualquier criterio. 
Estas referencias desarrollan un parámetro que se manifiesta únicamente a partir de la evaluación de la dignidad desde una perspectiva de las personas afectadas en sus derechos que no puede extenderse a otros individuos. No obstante, podrían constituir modelos en la actividad de la evaluación, conforme sostiene Humberto Nogueira Alcalá (2003): «los derechos personalísimos protegen a la persona en sí misma, con independencia de su relación con los demás» (p. 62), sobre la base de la teoría de Gregorio Peces-Barba (1994), respecto de la clasificación de los derechos según el criterio del bien jurídico protegido y su finalidad, que fundamenta la universalidad, progresividad e interdependencia de los derechos humanos (p. 613).

b) El metaprincipio de la primacía de la realidad, en la esfera laboral, exige que el caso evaluado sea determinado por el juez, quien examinará los hechos, las referencias sociales y laborales y analizará el contexto normativo.

Nótese que estas referencias resultan diferentes a la aplicabilidad de principios como la igualdad, el debido proceso, el acceso a la tutela judicial efectiva, que son los correlacionales para efectos de los objetivos del presente texto, porque estos admiten un patrón referencial aplicable a cualquier ámbito y referencia que, por ejemplo, no logra ser asumido en tal dimensión cuando participa un niño o un adolescente, dado que este no es sujeto procesal.

Por ello, es importante detallar que el interés superior del niño requiere de un punto de referencia para que pueda ser integrado a la evaluación de una situación, que no se da en cualquier etapa ni puede ejecutarse de modo discrecional, porque ocasionaría un daño o un error en su determinación, como actualmente sucede, y este es el principal elemento que permite identificarlo como un metaprincipio. 


\section{LA APLICABILIDAD DEL METAPRINCIPIO DEL INTERÉS SUPERIOR DEL NIÑO CON RESPECTO DE OTROS DERECHOS INTERDEPENDIENTES EN MÉRITO DEL BLOQUE DE CONVENCIONALIDAD}

Tomando en cuenta el elevado contexto de atomización del conflicto familiar por acción de la legislación sustantiva y procesal nacional (Bermúdez-Tapia, 2012, p. 163), en la cual se amplía el margen discrecional de los progenitores para actuar en forma negligente, desidiosa, maliciosa y temerariamente, los jueces deben considerar el carácter de interdependencia de los derechos del niño o el adolescente que participa en el trámite de un expediente judicial y, por tanto, cuando revisen un expediente judicial, deberán ejecutar una evaluación a partir del bloque de convencionalidad.

En este sentido, superamos el patrón de referencia del bloque de constitucionalidad, pues la tutela de los derechos de un niño o un adolescente en el trámite de un proceso judicial no es garantizada ni evaluada en función del conflicto humano en el cual se encuentra. Por tal motivo, planteamos un modelo basado en una estructura secuencial de acuerdo con el bloque de convencionalidad:

a) Se deben evaluar los elementos de hecho y de derecho expuestos en los petitorios de las partes procesales, de modo que el juez evalúe su impacto para evitar la ampliación del contexto negativo que asume el niño o el adolescente en el proceso judicial.

En este contexto, esta acción posibilita tutelar derechos en un ámbito individual, tales como los siguientes:

- Al desarrollo de la dignidad.

- A la igualdad ante la ley.

- A la identidad personal. 
- A comunicarse y solicitar información a la administración pública, aun siendo menor de edad.

- A la paz, a la tranquilidad, a gozar de un ambiente equilibrado y adecuado al desarrollo de su vida.

- A no sufrir ningún tipo de violencia.

- A la libertad y seguridad personal.

- A una asesoría legal, aun siendo menor de edad, considerando que, en los conflictos familiares, los mismos progenitores pueden atentar contra sus derechos anulando la representación legal que puedan ejercer.

- $\mathrm{Al}$ acceso a un servicio de impartición de justicia en forma gratuita.

- A analizar y criticar las decisiones judiciales, porque, sin ser parte procesal, pueden verse afectados por la decisión judicial.

b) Complementariamente, esta acción permite tutelar derechos que tienen, en sí mismos, una condición multidimensional (Carpizo, 2011), por la interdependencia de estos (Fernández Ruiz-Gálvez, 1997), para que pueda evaluarse un contexto basado en el análisis de derechos colectivos e intereses difusos, por ejemplo:

- A ser protegido como integrante de un grupo en situación de vulnerabilidad o estar en condición de riesgo.

- A vivir en un ambiente familiar, que es extensivo a todos los integrantes de una familia en crisis o en situación de división.

- A gozar de sus derechos, que involucran el acceso a servicios públicos como el de educación, salud o seguridad que puedan optimizarlos. 
- A tener la garantía de que se le brinde un debido proceso y una tutela judicial efectiva, pese a no ser parte procesal.

- A ser parte de una decisión que esté motivada, por cuanto su vida y desarrollo dependerá de dicha acción judicial.

- A estar bajo el alcance de la pluralidad de instancia judicial, en caso de que sus derechos puedan verse afectados por el conflicto que sostienen sus progenitores.

- A no ser privado del derecho de defensa en ningún estado del proceso, a estar informado del trámite judicial y tener una asesoría legal que, si bien está redactado para evaluarse solo a favor de las partes procesales, debe entenderse como extensivo a toda situación judicial sin importar la condición personal o procesal.

Mediante este esquema argumentativo, se supera la normatividad nacional, tanto por la aplicabilidad del Código del Niño y del Adolescente, el Código Procesal Civil, la Ley n. ${ }^{\circ} 30466$ y la Ley Orgánica del Poder Judicial, para ejecutar:

a) La acumulación de condiciones tuitivas a favor de los niños y los adolescentes respecto de otros derechos interdependientes derivados del ejercicio de la parentalidad de los progenitores, como la determinación de la tenencia y el régimen de visitas.

b) La admisión de la actuación discrecional del juez en la toma de decisiones extraordinarias para garantizar derechos de orden interdependiente $y$, sobre todo, para evitar situaciones que generen la omisión en la prestación de la obligación alimentaria o la sustracción de menores, en caso de que exista una situación de violencia elevada entre los progenitores.

Como se observa, el bloque de convencionalidad expuesto permite superar los parámetros que impone la legislación y la práctica judicial nacional (Rey, 2006), debido a que faculta la tutela 
inmediata, directa y proporcional de los derechos interdependientes de los niños y adolescentes, conforme con los tratados internacionales aplicables en estos casos sin que se ejecuten procesos judiciales paralelos, complementarios, derivados, secundarios, porque la atomización de pretensiones en procesos autónomos es perjudicial al elevar el conflicto familiar.

Sustentamos nuestro argumento, que amplía el margen deliberativo filosófico del alcance del interés superior del niño, en que el acto de ponderación, al implicar un procedimiento racional para la aplicación de las normas jurídicas o un subterfugio retórico — como anota Bernal Pulido (2006) - , sería perjudicial para la acción de tutela a favor de sujetos que no son parte procesal en un trámite judicial. Con ello la labor del juez adquiere una connotación superlativa, ya que deberá desarrollar su labor en función de la necesidad de velar no solo por la justicia, sino también porque un alimentista dependerá de su decisión y puede aplicar directamente la Convención Americana sobre Derechos Humanos (Martínez, 2017).

\section{EL DERECHO ALIMENTARIO COMO DERECHO MULTIDIMENSIONAL}

Los alimentos, como elementos de referencia sustantivos y procesales, no deben limitarse a un contexto vinculado al judicial en la especialidad de familia, dado que su valor es superior, pues poseen fundamentos constitucionales, procesales, civiles, penales y del derecho internacional de los derechos humanos. Por tanto, desde esta perspectiva, advertimos que el derecho alimentario es, a la vez:

a) Un derecho fundamental, ya que garantiza la supervivencia de un niño o adolescente, al ser una persona (o grupo) en situación de riesgo o vulnerabilidad. 
b) Un derecho humano, conforme se desprende de los tratados internacionales aplicables al ámbito del presente texto, de acuerdo con el bloque de convencionalidad desarrollado previamente.

c) Un derecho constitucional, conforme se infiere de la interpretación literal de la Constitución peruana de 1993.

d) Un derecho programático, porque involucra también la intervención del Estado, especialmente en la provisión de servicios públicos que garanticen el acceso a un sistema de impartición de justicia, y de provisión de alimentos.

e) Un derecho interdependiente, toda vez que no puede evaluarse en forma excluyente a otros derechos, conforme se indica en el tercer punto del presente texto (Rubio, 1989).

En esa línea, determinamos que el derecho alimentario es un derecho natural que se sustenta en la evolución de un criterio sociobiológico a un patrón referencial político que se traduce en una obligación y un derecho, todo ello sobre la base del análisis de la libertad (Caballero, 2006) y, por ello, es un derecho multidimensional e interdependiente con otros derechos. De este modo, resulta incongruente la continuidad de la práctica judicial que refiere el carácter excluyente de evaluar la obligación de prestar alimentos sin estimar la patria potestad, la tenencia, el régimen de visitas y la condición que determina las obligaciones y los derechos de los progenitores, de conformidad con la evaluación del bloque de convencionalidad.

\section{LA NECESARIA EVALUACIÓN DEL BLOQUE DE CONVENCIONALIDAD SOBRE LA LEGISLACIÓN NACIONAL}

En el trámite de un proceso judicial, los derechos de los niños y los adolescentes son relativizados frecuentemente; demostramos esta afirmación con los siguientes ejemplos: 
a) El período temporal entre el inicio del conflicto familiar y la sentencia judicial suele acreditar la afectación sistemática de sus derechos, e inclusive los derechos del progenitor que tiene a cargo su cuidado, por acción del otro progenitor.

b) La evaluación del derecho, tanto en un recurso cautelar como en una medida de tutela inmediata - según sea el caso-, no condiciona el nivel de violencia y desatención generado por el progenitor que provoca el daño o la omisión a prestar alimentos.

c) La complementación de dos procesos judiciales seguidos (alimentos y delito de omisión de asistencia familiar) refleja que dos magistrados, en dos momentos temporales, no logran ser eficientes en la atención de un derecho natural.

d) Los atentados contra otros derechos no son evaluados por los progenitores ni por el sistema de impartición de justicia, al nivel de que en el Tribunal Constitucional se ha generado jurisprudencia sumamente importante sobre la base de la progresividad de derechos (Bermúdez-Tapia, 2019c, pp. 219-270).

e) La legislación no puede atender de forma eficiente la tutela de derechos de niños y adolescentes, especialmente porque asigna parámetros ajenos a la realidad familiar, por ejemplo, la conciliación, que, tanto en su procedimiento como en sus efectos, resulta estéril (Bermúdez-Tapia, 2019b, pp. 187-201).

f) El análisis del proceso judicial exige la evaluación formal de las acciones de las partes procesales, por lo que los niños y los adolescentes se encuentran condicionados a una participación pasiva, secundaria e incluso invisible.

A partir de esta situación, conviene tener en cuenta la potencial evaluación del bloque de convencionalidad en la práctica judicial, 
para que así se puedan inaplicar algunos parámetros normativos que limitan la acumulación procesal de pretensiones, cuando se trata del mismo conflicto familiar, porque solo de este modo se ejecutaría una acción diligente, temporalmente eficiente y tuitiva.

Fundamentamos nuestra posición sobre la base del argumento que desarrolla David Andrés Murillo Cruz (2016), quien sostiene el desarrollo de un método dialéctico entre el bloque de convencionalidad en su relación con el bloque de constitucionalidad en el Sistema Interamericano de Derecho Humanos, porque, en esencia, la «fundamentación por aproximación conceptual» y teleológica del primero se sustenta en el segundo. Este criterio es ampliado por Constancia Núñez (2015), quien señala que los conceptos de «bloque de constitucionalidad», "control de constitucionalidad» $\mathrm{y}$ «control de convencionalidad» están ligados (p. 158), pero, por su naturaleza jurídica, intervienen en diferentes niveles.

En este orden de las cosas, la diferenciación parte del análisis de la naturaleza jurídica y el origen de la legislación en cada ámbito, por cuanto:

a) El bloque de constitucionalidad posibilita desarrollar normas no contenidas en la Constitución de un Estado, pero con ella guarda relación material, filosófica y política, en mérito principalmente del traslado de «cuotas de libertad» de parte de los ciudadanos a favor del Estado, respecto del poder constituyente que permite construir un marco normativo sobre el cual se detalla la legitimidad y la legalidad de la administración pública para realizar sus funciones en mérito del poder que se le ha conferido (ius imperium).

b) El bloque de convencionalidad equivale, en lo procedimental, al bloque de constitucionalidad; sin embargo, se efectúa en una referencia del ámbito internacional sobre la cual los Estados 
actúan como entes que ceden una cuota de su soberanía para la materialización de un orden normativo internacional, mediante el cual se ejercen los derechos humanos, los derechos fundamentales, los derechos constitucionales $\mathrm{y}$ la legislación, en general, tanto a nivel internacional como nacional, con lo cual adquiere un papel superior al bloque de constitucionalidad.

De este modo, los magistrados - especialmente los que se ocupan de la justicia de paz y justicia especializada- podrían aplicar un criterio teleológico superior que reduzca el margen de violencia que asume un niño o un adolescente en el trámite de un proceso judicial provocado y desarrollado por sus progenitores, en el cual no puede intervenir ni posee un margen de decisión sobre sus propios derechos, tomando en cuenta que estos son autónomos de los derechos e intereses de los progenitores.

Consideramos que este planteamiento mejoraría la calidad en la gestión de un conflicto familiar en el contexto judicial y garantizaría un nivel de tutela idóneo para los derechos de sujetos ajenos a las partes procesales, en función de valores superiores, con lo cual el metaprincipio del interés superior del niño se despojaría de su condición abstracta e indefinida para convertirse en un instrumento eficiente no solo frente al trámite del proceso, sino también frente a la ley que continúa deshumanizando el contexto familiar por establecer un parámetro equivalente a la evaluación de un conflicto civil puro. 


\section{REFERENCIAS}

Aguilar, G. (2008). El principio del interés superior del niño y la Corte Interamericana de Derechos Humanos. Estudios Constitucionales, 6(1), 223-247.

Alexy, R. (1993). Teoría de los derechos fundamentales. Madrid: Centro de Estudios Constitucionales.

Bermúdez-Tapia, M. (2011a). Derecho procesal de familia. Lima: San Marcos.

(2011b). La constitucionalización del derecho de familia. Lima: Ediciones Caballero Bustamante.

(2012). Derecho procesal de familia. Lima: San Marcos. (2017). El amparo y la tutela de derechos en conflictos familiares judicializados. En Carpio, E. y Sáenz, L. (coords.), $E l$ amparo en la actualidad. Posibilidades y límites (pp. 203-221). Lima: Tribunal Constitucional del Perú y Colegio de Abogados de Lima.

(2019a). Elementos procesales y probatorios en el derecho de familia. Bogotá: Ediciones Nueva Jurídica.

(2019b). La aplicabilidad del control difuso en la evaluación del acta de conciliación sometida a proceso de ejecución, en caso se atente contra los derechos de niños y adolescentes involucrados. En Pontificia Universidad Católica del Perú, II Congreso Iberoamericano Interdisciplinario de la Familia e Infancia: violencia contra niños, niñas y adolescentes en colectivos vulnerables (pp. 187-201). Lima: Pontificia Universidad Católica del Perú.

(2019c). La evaluación constitucional de derechos en el derecho de familia. Lima: Gaceta Jurídica.

Bernal, C. (2006). La racionalidad de la ponderación. Revista Española de Derecho Constitucional, (77), 51-75. 
Burgorgue-Larsen, L. y Úbeda, A. (2011). The Inter American Court of Human Rights. Case Law and Commentary. New York: Oxford University Press.

Caballero, J. (2006). La teoría de la justicia de John Rawls. Iberofórum. Revista de Ciencias Sociales de la Universidad Iberoamericana, 1(2), 1-22.

Carpizo, J. (2011). Los derechos humanos: naturaleza, denominación y características. Cuestiones Constitucionales, (25), 3-29.

Corte Interamericana de Derechos Humanos (1999). Caso Castillo Petruzzi y otros vs. Perú. Recuperado de https://www.corteidh. or.cr/docs/casos/articulos/seriec_52_esp.pdf (2001). Caso Ivcher Bronstein vs. Perú. Recuperado de https://www.corteidh.or.cr/docs/casos/articulos/Seriec_84_ esp.pdf

(2018). Caso Ramírez Escobar y otros vs. Guatemala. Recuperado de http://www.corteidh.or.cr/docs/casos/articulos/ seriec_351_esp.pdf

Fernández Ruiz-Gálvez, M. (1997). Derechos humanos: ¿yuxtaposición o integración? Anuario de Filosofía del Derecho, (14), 679-702.

Fortin, J. (2005). Children's rights and the Developing Law. London: Cambridge University Press.

Hewitt-Taylor, J. (2008). Providing support at home for children and young people who have complex health needs. Chichester: John Wiley \& Sons.

International Human Rights Law Clinic, Chief Justice Earl Warren Institute on Race, Ethnicity and Diversity \& Immigration Law Clinic (2010). In the child's best interest? The consequences of losing a lawful immigrant parent to deportation. Berkeley: Berkeley Law University of California. 
Krauss, D. y Sales, B. (2001). The child custody standard. What do twenty years of research teach us? En White, S. (ed.), Handbook of youth and justice (pp. 411-435). New York: Kluwer Academic Plenum Publishers.

Lopera, G. (2004). Los derechos fundamentales como mandatos de optimización. Doxa. Cuadernos de Filosofía del Derecho, (27), 211-244.

Martínez, A. (2017). ¿Derecho supranacional o derecho convencional? Importancia de su determinación. Revista Jurídica Valenciana, (33), 31-47.

Murillo, D. (2016). La dialéctica entre el bloque de constitucionalidad y el bloque de convencionalidad en el Sistema Interamericano de Derechos Humanos. Revista de Derecho Público, (36), 1-35. Recuperado de https://derechopublico.uniandes.edu.co/ components/com_revista/archivos/derechopub/pub569.pdf

Nogueira, H. (2003). Teoría y dogmática de los derechos fundamentales. Ciudad de México: UNAM.

Núñez, C. (2015). Bloque de constitucionalidad y control de convencionalidad en Chile. Anuario de Derechos Humanos, (11), 157-169.

Peces-Barba, G. (1994). La universalidad de los derechos humanos. Doxa. Cuadernos de Filosofía del Derecho, (15-16), 613-633.

Peczenik, A. (1983). Grundlagen der juristischen Argumentation. Viena/Nueva York: Springer.

Polaino-Lorente, A. (1988). Las depresiones infantiles. Madrid: Morata.

Rey, E. (2006). El bloque de constitucionalidad. Aplicación de tratados internacionales de derechos humanos. Estudios Constitucionales, 4(2), 299-334. 
Rubio, F. (1989). El bloque de constitucionalidad. Revista Española de Derecho Constitucional, (17), 9-37.

Sheehan, J. (2018). Family conflict after separation and divorce. London: Palgrave.

Suárez, J. (2006). Crítica a la razón en la filosofía del siglo XX. Antioquía: Universidad de Antioquía.

Thomlison, B. (2010). Family assessment handbook: an introduction and practical guide to family assessment. Belmont: Thomson Higher Education. 